\title{
Characterization and Prediction of Protein Interfaces to Infer Protein- Protein Interaction Networks
}

\author{
Ozlem Keskin*, Nurcan Tuncbag and Attila Gursoy
}

Koc University, Center for Computational Biology and Bioinformatics, and College of Engineering, Rumeli Feneri Yolu, 34450 Sariyer Istanbul, Turkey

\begin{abstract}
Complex protein-protein interaction networks govern biological processes in cells. Protein interfaces are the sites where proteins physically interact. Identification and characterization of protein interfaces will lead to understanding how proteins interact with each other and how they are involved in protein-protein interaction networks. What makes a given interface bind to different proteins; how similar/different the interactions in proteins are some key questions to be answered. Enormous amount of protein structures and experimental protein-protein interactions data necessitate advanced computational methods for analyzing and inferring new knowledge. Interface prediction methods use a wide range of sequence, structural and physico-chemical characteristics that distinguish interface residues from non-interface surface residues. Here, we present a review focusing on the characteristics of interfaces and the current status of interface prediction methods.
\end{abstract}

\section{A. PRINCIPLES OF PROTEIN-PROTEIN INTERACTION}

Proteins rarely act in isolation; rather they function in the crowded medium of other molecules and proteins. Protein interactions are central to structural and functional organization of the cell in vivo and underlie many biological processes, such as metabolic control, protease inhibition, DNA replication and transcription, cell adhesion, hormonereceptor binding, the action of antibody against antigen, intercellular communication, signal transduction, and regulation of gene expressions in cells. They also relate to allosteric mechanisms, to turning genes on and off and to drug design. Thus, understanding biological processes relies on a comprehensive knowledge of different types of proteinprotein interactions and interaction mechanisms [1].

The study of protein interactions has two main goals: identifying interaction sites of proteins and predicting which proteins interact with which others. Recently, with the development of genomic technologies, massive amounts of data about protein interactions are being generated at an unprecedented pace. Studies using high-throughput experimental approaches such as yeast two-hybrid based methods [2, 3], mass spectrometry [4] and proteome chips [5] have each resulted in the accumulation of protein-protein interaction data. This extensive increase of protein interactions cover organisms from bacteria to eukaryotes and help to elucidate the large-scale organization of cellular processes [6]. Nevertheless, each method has its shortcomings. Most notably, all the methods suffer from a substantially high rate of falsepositives, and false-negatives. In addition to this, different methods are sensitive to different types of interactions that they can detect, hence the results are generally known to be

*Address correspondence to this author at the Koc University, Center for Computational Biology and Bioinformatics, and College of Engineering, Rumeli Feneri Yolu, 34450 Sariyer Istanbul, Turkey;

E-mail: okeskin@ku.edu.tr biased towards certain protein classes [7]. In addition to the experimental methods, a growing number of computational methods for predicting protein interactions are emerging as reviewed below. These techniques exploit the already present genomic, structural and protein interaction data to infer new interactions in silico.

Collections of interactions among proteins form a complex interaction network (interactome) in the cell. The more we know about proteins, the more we see them as parts of complex networks or pathways rather than isolated entities. The function of a protein can be viewed by its position within this cellular interaction network $[8,9]$. Furthermore, the topology of the protein interaction networks may reveal the cell's higher-level functional organization [10]. For example, a key feature of biological systems is their robustness, which refers to the system's ability to respond to changes in the external or internal conditions successfully so that it will continue its basic functions. Biological systems are found to be amazingly resistant to damages in its components. In the case of protein-protein interaction network, topology of the network is found to be responsible for its robustness [11]. Further, investigation of protein-protein interaction maps show that some proteins are highly connected to others (acting as hub proteins), whereas some others have a few interactions (at the edges of the map) [12,13]. Scale-free characteristics of networks make them very resilient against accidental failures: even if $80 \%$ of randomly selected proteins fail, the remaining $20 \%$ still continue to carry out the cellular functions [10]. However, protein networks with hubs are shown to be vulnerable to systematic attacks at highly connected proteins. In addition, these proteins are found to be essential by experimental studies [14]. A balance of specificity and promiscuity of interactions is needed for being an edge or hub protein. Integrating structural knowledge can have a significant role in rationalizing the promiscuity/specificity of hubs, as well as other abstract properties of the network. For example, it is now known that some interaction sites are specific to bind to a single protein. On the 
other hand, it may be as well expected that other proteins may bind several different molecules at the same or overlapping locations [15-18]. Otherwise, it would be impossible for a protein to interact with tens of other proteins [19]. In a recent study, structural modeling and interface knowledge are combined with network analysis. Their results revealed that structural quantity, i.e., the number of distinct binding interfaces is a result of relationships between network topology and genomic features (e.g., hubs tending to be essential proteins) [16]. Knowledge of protein interfaces provides not only understanding of the relations among the protein and its surrounding and function but also engineering new interactions. Further, these interfaces may be used to identify drug targets, design drug-like peptides to inhibit unwanted interactions.

\section{Proteins Interact Through their Interfaces}

Protein-protein interactions occur at the surface of a protein and are biophysical phenomena, governed by the shape, chemical complementarity, and flexibility of the molecules involved as well as the environmental conditions. The physical binding of protein structures occur through weak, noncovalent interactions. The particular region where two protein chains come into contact is termed a binding site or an interface. Towards the common goal of understanding how proteins interact, a number of studies have characterized the properties of interfaces between proteins [20-26]. These interface regions generally resemble to the cores of globular proteins; they are usually comprised of residues with nonpolar or aromatic side chains [27, 28]. As an example, Fig. (1) shows the interface present between $\alpha$ - and $\beta$-chains of the human hemoglobin (PDB ID: 1yhe). Protein interfaces have long been studied at both the protein level and the domain level. They have been represented as interface data sets and deposited into databases to be used in identification of general properties of them. Some of these databases are SCOPPI [29], CBM [30], InterPare [31], 3DID [32], PIBASE [33], ProtCom [34]and PRINT [35, 23]. Physical and chemical properties of interfaces can be studied using these databases. Interface characteristics and available databases will be discussed in the following section.

\section{Characteristics of Protein Interfaces}

Characteristics of the protein-protein interfaces are crucial to elucidate binding principles and provide clues about the binding site prediction algorithms. One of the most important aims in interface analysis is to identify properties which may distinguish binding parts from the rest of the protein surface.

\section{Physical and Chemical Properties of Interfaces}

Interactions have been studied on a large number of complexes by numerous groups [15, 20-23, 25, 27, 36-49]. In general, interfaces tend to be planar or well packed depending on the type of interaction $[15,21]$. The residue composition usually differs for those complexes that are transient versus those that are obligate. This is probably due to different strength of interactions between the proteins, the former relying more on salt bridges and hydrogen bonds, whereas the latter rely more on hydrophobic attractions [22, 45]. There are also many examples of both geometric and electrostatic complementarity between the two sides of interfaces [45, 46, 50, 51]. Jones and Thornton (1997) have considered 59 different protein-protein interfaces which were classified into four groups; homo-dimers, enzyme-inhibitor, antibody and hetero complexes. They stated that homodimers are more hydrophobic and they have larger interfaces. Hetero-complexes are less hydrophobic than homo-dimers. Small interfaces prefer to interact through small pockets to exclude solvent. In spite of detecting significant differences, they concluded that there is not a strict pattern to identify different types of protein-protein interactions [52]. Lo Conte et al (1999) have also demonstrated the effect of the interface size in a similar manner and they stated that the standard sized $\left(1600 \pm 400 \AA^{2}\right)$ interfaces are more stable and permanent interfaces are closely packed like the interior of the pro-

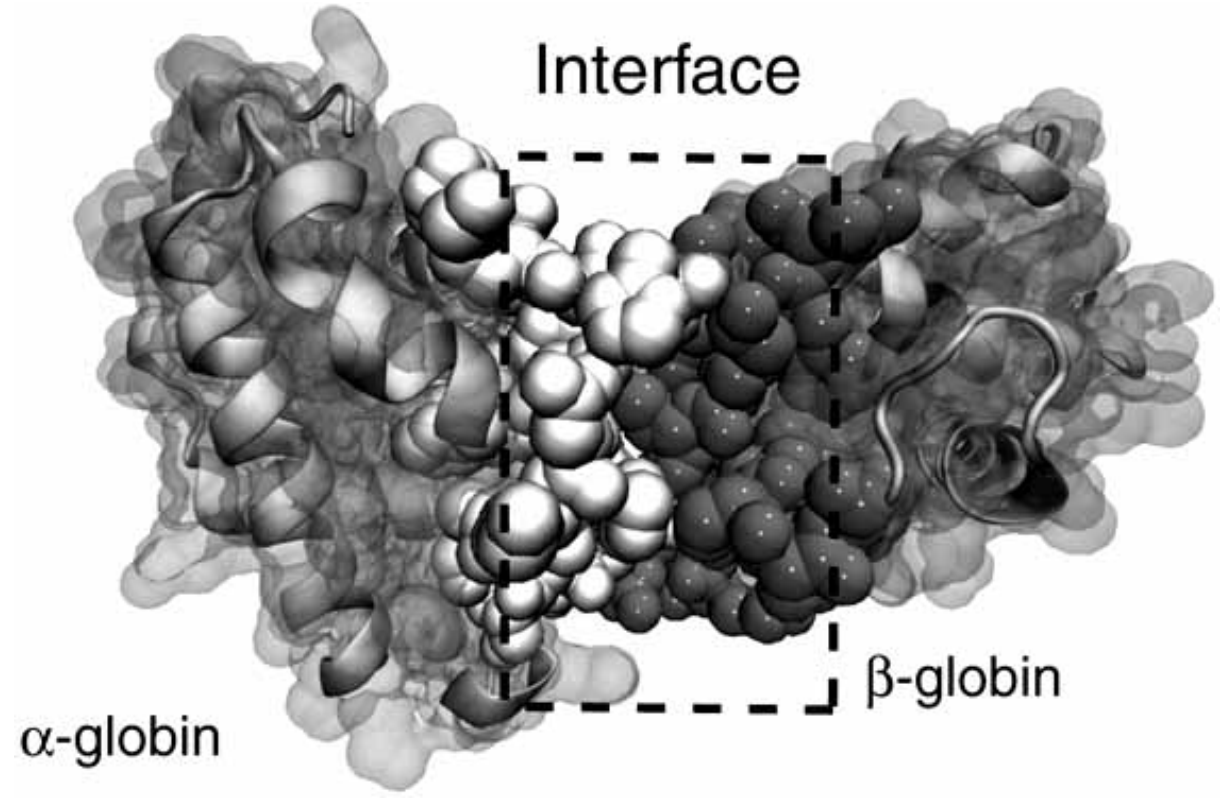

Fig. (1). Illustration of the protein - protein interfaces. The interface region between $\alpha$-globin and $\beta$-globin is illustrated in sphere representation, the rest of the chains is in ibbon diagram. The interface region is enclosed with the dashed box. 
teins [45]. Larsen et al. (1998) investigated a dataset of 136 homodimeric complexes and signified that one-third of the interfaces have a distinguishable large hydrophobic core, whereas the remaining interfaces have smaller hydrophobic patches with polar contacts and water mediated interactions. Amino acid frequencies in the interface region are also an important parameter in characterization of these regions [53]. Using only amino acid composition and residue-contact preferences, Ofran and Rost (2003) have reached 63-100\% accuracy to predict interaction types [54]. Additionally, interface regions are rich in aromatic and hydrophobic clusters. The residue propensity of the interface regions is found to be similar to the interior of the proteins [20]. Hydrogen bonds form the electrostatic interaction between the monomers which are found frequently in transient interactions [55]. Also, despite their rare occurrence, disulphide bonds have large contribution on stabilization of the binding [56].

\section{Conservation of the Interfaces}

Evolutionary conservation of the residues is an important property of the interface regions. Residues in the interface region are thought to be evolving at a slower rate when compared to surface residues. The importance of conservation in protein-protein interfaces is well studied [57-59]. Interface residues are found to be slightly more conserved than the rest of the surface residues [57, 59]. Caffrey et al. analyzed interfaces using surface patches, they found that the difference between the patches and the rest was even less pronounced [57]. Further, buried interface residues in obligate interfaces were observed to be more conserved than partially buried interface residues.

Tools to extract conservation profiles of surface residues can be very helpful for large-scale characterization of functional regions in proteins. ConSurf $[60,61]$ is a web server which incorporates several phylogenetic based algorithms [62] to estimate conservation scores of surface residues given 3D structures. Using ConSurf, examination of protein surfaces were revealed to have patches of highly conserved residues which are functionally important.

\section{Hot Spots in Protein Interfaces}

One of the hottest topics in protein interfaces is "hot spots'. Binding energies are not uniformly distributed along the binding sites. Although the interfaces can be quite large, it was shown in some systems that only a small fraction of the residues contribute to the majority of the binding energy [38]. These critical residues are called 'hot spots'. Alanine Scanning Mutagenesis is an experimental method to identify these residues which is based on the fact that if a residue has a significant drop in binding affinity when mutated to alanine then it is a hot residue $[38,63]$. Due to limited availability of experimental data about hot spots, researchers focused on computational methods to introduce several approaches to detect them [64]. Some of them have developed energy based methods to predict hot spots [65-67]. Molecular dynamics studies have also been used to investigate the energetic contributions of interface residues [68,69]. These methods, though quite accurate, are time consuming. As an alternative to energetics of residues, conservation is another important property to detect hot spots. Structurally conserved residues and hot spots are found to be correlated with each other significantly $[28,40,70]$. These hot spots are also found to be buried and tightly packed with other residues resulting in densely packed clusters of networked hot spots, called 'hot regions' [40]. Residue conservation alone is not sufficient to identify hot spots. Hot spots are buried and some amino acids are seen frequently as hot spot; such as Arg, Tyr and Thr [27]. HotSprint, another conservation based method, combines these three properties (conservation, solvent accessibility (ASA), residue propensity) to detect hot spots with an accuracy of 76\% [71]. Hot spots are more conserved and have similar physico-chemical properties: these observations can be used to distinguish interacting regions from the rest of the surface [72].

\section{Structural Aspects of Interfaces}

The origin of the affinity of protein-protein complexes, stability of super-assemblies and, the specificity of interactions between proteins are still not very well understood. In addition to the covalent bonds that stabilize each chain of the complex, long-range interactions are predominant in the stability and specificity of interfaces. These are electrostatic interactions, hydrogen bond interactions, van der Waals attractions and repulsions and hydrophobic forces. In order to maximize the effect of these forces, a good shape complementarity is necessary. It is known that in order to have a stable complex, the two molecules should have complementary surfaces, as in the key and lock model. The protein interfaces may change in size. Their sizes are measured as the change in their solvent accessible surface area ( $\triangle \mathrm{ASA})$ when going from a monomeric to a dimeric state. On average, interfaces bury $1600 \AA^{2}$ with similar chemical character close to the average protein surface, and involve nine hydrogen bonds and the same packing density as the protein interior [45].

\section{Pockets in Interfaces}

The protein interfaces are not flat but rather filled with pockets, crevices, and indentations [73]. Pockets in protein interfaces may be either unfilled or complemented. Unfilled pockets represent packing defects in the protein interfaces. They are the cavities occurring after protein-protein association and remain unfilled by the companying protein partner. On the other hand, complemented pockets are defined to be present when the two proteins are separated, but disappear following association and represent tightly fit regions [24]. It is observed that hot spot residues prefer to sit in complemented pockets, and are disfavored in unfilled pockets. Interestingly complemented pockets often pre-exist binding. For 18 protein-protein complexes with complemented pockets whose unbound structures were available, in 16 the pockets were identified to pre-exist in the unbound structures [24]. Size and shape of pockets and distribution of functional groups in these pockets are critical to design selective molecules or peptides to act as drugs.

A variety of computational methods are available to detect pockets on protein surfaces. Most of them use geometric criteria to find clefts and cavities. SURFNET identifies voids, internal cavities and pockets between molecules by fitting virtual spheres between the molecules [74]. The program CAST uses alpha shapes and triangulations of the protein surface to detect cavities and pockets $[73,75]$. PASS 
uses an iterative procedure to locate the probe spheres to identify the shape of the surface [76]. POCKET identifies the pocket and cavities by using rectangular grids and denotes grid points as either solvent accessible or inaccessible [77]. PocketPicker is an automated grid based technique that specifies the shape of a potential binding site with regard to its solvent accessibility [78]. Pocketfinder is another algorithm based on the transformation of Lennard Jones potentials calculated from the three dimensional structure of the protein [79].

\section{Flexibility of Interface Residues}

The binding site of an unbound monomer (prior to binding) is enclosed by more water molecules and has less flexibility as depicted by low temperature factors compared to the rest of the surface [56]. Similarly, Cole and Warwicker (2002) have showed that the interfacial residues are less flexible than the rest of the protein surface [80]. Further, some critical residues in interfaces are found to be less flexible compared to the rest of the protein surface and interface residues [69, 81, 82]. These residues are highly packed and form clusters among themselves [40]. They are observed to form pre-organized binding motifs at protein interfaces even in the unbound cases. This organization might be to minimize the entropic cost upon complex formation.

\section{Specific and Promiscuous Interfaces}

Some binding sites are specific whereas others are utilized to bind to several different proteins. It is recently shown that most proteins interact with few other proteins, as a small but significant proportion of proteins, the 'hubs', interact with many other proteins $[83,84]$. Since the surface areas of proteins are limited, one would expect that some interaction sites on the protein surface might be re-utilized at different times as well as some binding sites are distinct. If the same site is used, the binding to each partner should be at different times whereas if different sites are used to bind to different proteins, these associations might be simultaneously. A

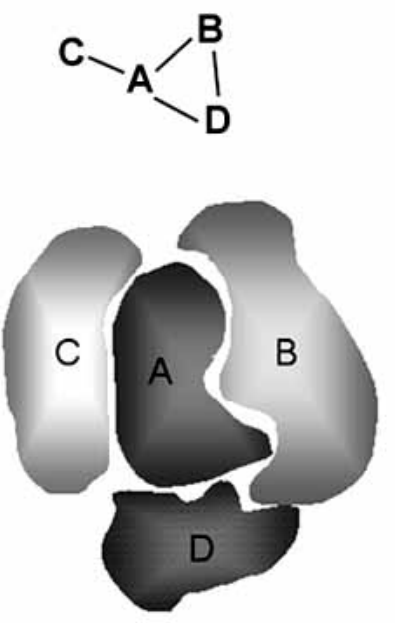

schematic representation is provided in Fig. (2). Structural knowledge of proteins is critical to identify the multiply used sites.

For example, crystal structures of the Elongin B/Elongin $\mathrm{C} / \mathrm{VHL}$ and Elongin B/Elongin C/SOCS2 [85, 86] complexes provide such clues. The concept of functional switches in transcriptional regulation [87] was emphasized by Beckett, focusing on the ability of proteins to bind alternative proteins at the same binding site. Our previous analysis highlights that there exist conserved interactions of a given site when interacting with multiple partners. This means that even though the partner proteins are different there are some critical residues in the binding site that make conserved interactions with multiple proteins [15]. Thus, while the patterns of the local interactions are similar in multi-partners and in single-partners, the multi-partners have been optimized by evolution to accommodate different ligand shapes, sizes and composition.

\section{Artificial Interfaces from Crystal Complexes}

If two proteins interact with each other in vivo, then they form a biological complex, and their interaction is formed through a biological contact. However, sometimes, the entries in the Protein Data Bank (PDB) [88], have artifacts of crystallization meaning that some of the complexes there would not occur in solution or in the physiological state. Determining which contacts are biological and which are not is often difficult [89].

There are some ways to differentiate biological contacts from non-biological ones. Several groups have successfully used conservation scores to predict biological proteinprotein binding sites $[89,90]$ which conclude that conservation in combination with other factors can accurately discriminate homodimers from crystal contacts. Further, interface size is an important characteristic in distinguishing crystal and biological complexes [20,89,91]. A threshold of 400 $\AA^{2}$ was used in protein quaternary structure (PQS) for identi-

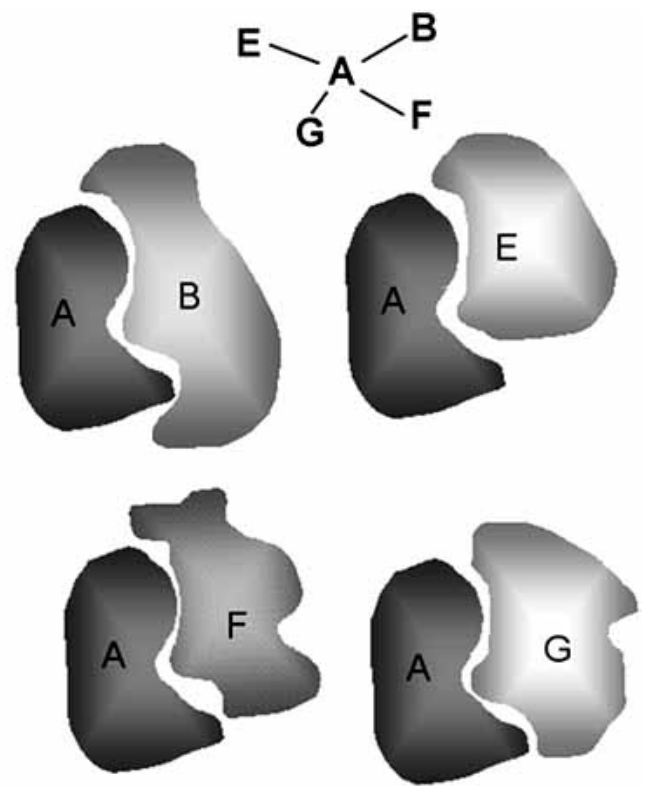

Fig. (2). An interface can be specific (left panel). The interaction between proteins A and B, C, D take place through different interfaces. On the other hand, an interface can be used to bind to several different proteins (right panel). The same interface on Protein A is used to bind to proteins B, E, F and G. 
fying biological complexes [92]. Similarly, when the number of interface residues was less than 10 , the interface was considered to result from the crystal packing rather than biological inter-subunit interactions [23, 47]. Schoemaker et al. generated a set of conserved domain-domain interactions by structural alignment to distinguish the biologically relevant interactions. According to these conserved modes, they reached an accuracy of $90 \%$ on all globin interacting pairs without false positives [30]. Another significant factor to distinguish artificial interfaces is amino acid frequency on the interaction site. Carugo et al. (1997) stated that if the binding site amino acid composition is similar to the rest of the surface of that protein, then this interaction is possibly crystal packing [93]. In addition, multiple features can be also combined for predicting biological complexes using machine learning approaches as in Oxclass [94]. To characterize protein interaction types, they used six different interface properties: Interface area, interface area ratio, amino acid composition, correlation between surface and interface regions, gap volume index, and conservation score of the interface. Using a combination of the properties in a support vector machine application, NOXclass distinguishes biological and non-biological interfaces reaching an accuracy of $91.8 \%$ based on three parameters (interface area, interface area ratio and area based amino acid composition).

\section{Prediction of Protein-Protein Interfaces}

Experimental detection methods have so far yielded a considerable amount of data on protein-protein interaction and their relative strengths. On the other end of the spectrum, computational prediction methods can address proteinprotein interaction problem at different levels from a different perspective. They may focus on in depth analysis or carry out a broad scale analysis across large datasets to identify putative interactions.

Various approaches towards determining residues that are part of protein interfaces adopt two general viewpoints: protein sequence (amino acid composition and their specific order) and protein structure (3D spatial orientations of amino acids). Some take into account common physico-chemical and geometric characteristics of interfaces of interacting proteins. Both viewpoints highly make use of the signatures evolution is known to leave on interacting proteins. Below we provide a brief review of different studies using these approaches.

\section{Prediction when Structure is not Available}

Predictions can be performed by using purely sequence information. For example, Res et al., 2005 [95] applied the support vector machines (SVMs) using residue composition and evolutionary information. The residue composition is calculated by the corresponding column of the target residue in the Multiple Sequence Alignment (MSA). This feature is calculated for the target residue and its 8 neighbors in a 9residue sequence window. The prediction performance is measured by leave-one-out cross validation method at a success rate of $64 \%$ [95].

Amino acid composition is found to be different in interfaces of transient complexes compared to other protein interfaces. Therefore, using sequence information alone can dif- ferentiate interface types successfully [54]. In a recent work, Ofran and Rost introduced a machine learning based prediction method which only considered sequence information. They derived the binding site features from 3D structures during training, but in the prediction step they only consider the sequence information. The neural network (NN) is trained on windows of 9 sequentially consecutive residues and if any atom of the central residue in this window is in contact with the any atoms in other protein, the window is identified as a binding site. Their average accuracy is $61 \%$ covering $90 \%$ of the proteins in their test set [96]. In a recent study, non-gapped sequence segments were used to predict interacting residues [97]. The results revealed that continuous sequence segments (of length nine) are present in protein-protein interfaces with a ratio of $37 \%$ of the complexes in their dataset. Their prediction accuracy ranged between $10-60 \%$ depending on benchmarking and scoring scheme used.

\section{Prediction when Structure is Available}

As the structural data become available, more studies with features derived from structures appeared in recent years. These features include patch analysis, accessible surface area, simplified surface and potentials, spatial neighbors, 3D motifs, and hotspots. Jones and Thornton's work [52] was one of the earliest studies that utilized structural information to predict binding site location. Solvation potential, residue interface propensity, hydrophobicity, planarity, protrusion and accessible surface area from a dataset were used to find the patches on protein surfaces. They reached a success rate of $66 \%$ (39/59 structures). Zhou and Shan [98] extended Jones and Thornton's approach to predict individual binding site residues. Sequence profile and solvent exposure of surface residues with their 19 spatial nearest neighbors as prediction parameters were used to train a neural network. The training set included 744 pairs of interacting proteins which contain 564 homodimers and 180 heterodimers from PDB. In most cases, the predicted residues are observed to be distributed uniformly across the interfaces and a prediction accuracy of $70 \%$ was achieved. Fariselli et al. (2002) have also proposed a neural network based method to predict binding site residues. The neural network is fed with an 11 residue long window containing the target residue and its 10 spatial neighbors on the surface patch. The conservation was included as residue frequencies calculated by multiple sequence alignment. The prediction method reached an accuracy of $73 \%$ in a set of 229 heterodimers. To show the usefulness of the method, they predicted 3 binding site of Dnak molecular chaperone system, and found close agreement with experimental results [99]. Bradford et al. [100] used SVM utilizing the same set of surface properties of Jones and Thornton to asses the performance of SVM. They reached a success rate of $72 \%$. With a different training dataset containing 180 transient and obligate proteins, they achieved $76 \%$ accuracy. It was surprising that their method performed equally well both for transient and obligatory complexes in contrast with previous patch analysis that used separate scoring functions for different interaction types [52]. When they used transient interactions for training and obligatory ones for testing or vice versa they obtained similar results. They suggested that transient and obligate complexes share similar properties. In a recent study, crystallo- 
graphic temperature factors to account for the structural flexibility were integrated into SVM together with sequence and conservation attributes [101]. The surface residue and its spatial 12 neighbors with their features were used as input. They stated that structural flexibility alone was not sufficient but combining it with conservation increased the prediction performance.

The discriminating power of relative solvent accessible area (RSA) has been shown by a recent study [102]. RSA is defined as the ratio of the solvent exposed surface of a residue to maximum solvent exposed surface area for the same kind of residue. RSA is reported to improve predictions in addition to conservation and other common structural properties. A large size of training set was used in this work. An initial 1695 representative protein complexes from PDB were filtered by eliminating crystal and sequence redundant chains resulting in 435 chains. Authors used several machine learning algorithms including neural networks, SVM, and Linear Discriminant Analysis (LDA) to test the discriminative power of RSA combined with other frequently used features. Accuracy with RSA feature was reported to be around $74 \%$, whereas without RSA is $70 \%$. NN and SVM performed equally while LDA performing slightly worse [102].

\section{Other Approaches}

The studies reviewed above used mostly supervised learning algorithms. However, statistical/probabilistic and clustering based methods have been applied as well. Neuvirth et al. (2004) developed an empirical scoring function to distinguish between interacting and non-interacting residues. They focused on the heteromeric transient protein-protein interactions and extracted interface characteristics such as amino acid propensities, pairwise amino acid distribution, residue conservation, geometric properties and temperature factors and water molecules surrounding the binding site. An optimization step was performed to find the best combination of these parameters. The prediction algorithm, called ProMate, reaches a success rate of $70 \%$ where success is defined as at least half of the predicted interface residues should be contained in the real interface [56]. Another empirical scoring approach (PINUP) with linear combination of side chain energy score, conservation score and residue interface propensity found that energetic contribution of interface residues are distinctively different than the surrounding residues [103].

InterProSurf is a method based on patch analysis and clustering. A set of 72 complexes from PDB are used to find residue propensities for being on the interface or the surface of complexes. Interacting residues were predicted according to their accessible surface area and propensity characteristics. A clustering algorithm is used to group residues with high interface propensity. An accuracy of $70 \%$ was reached [104]. Hsu et al. [105] find clusters of conserved residues (that might correspond to hot regions [40]) from only sequence information using sequential pattern mining. Typically, conserved residues are found by using evolutionary information coming from multiple sequence alignments of homologous sequences. Authors follow a different approach, finding frequently occurring residue segments (patterns) separated by irregular and long gaps. They succeeded to show that conserved blocks (patterns) tend to cluster in space and are related to interface areas.

Clefts in protein surfaces can be used to predict binding hot spots [106]. Their results showed that the desolvation of clefts is a common characteristic across different interface types; and it is more discriminative than electrostatics to differentiate interfaces from other surface regions. In general, protein-ligand interfaces were predicted with a high level of prediction accuracy (93\%). Prediction success was found to be lower for protein-protein interfaces except enzymes where surface conservation seems to be the best predictor. Bayesian Networks are probabilistic graphical methods suitable for learning from incomplete datasets. Bradford et al. [107] trained Bayesian networks using several surface properties such as ASA, hydrophobicity from a nonredundant set of protein complexes. They tested different methods on a test set of 180 proteins. The results revealed a success rate of $82 \%$ which is $6 \%$ more than their previous SVM based method [100].

Most of the methods discussed above are available as web servers (listed in Table 1). A comparison of some servers are assessed in a recent study [108]. At a coverage of $50 \%$, the accuracies of cons-PPISP, Promate, PINUP, PPIPRED, SPIDDER, and meta-PPISP are $36 \%, 38 \%, 48 \%$, $27 \%, 33 \%$, and $50 \%$, respectively.

\section{No Single Feature is Discriminative Enough}

As it can be seen in the reviewed prediction approaches, no individual feature is reported discriminative enough. Adding more surface properties improves prediction results. Therefore, most binding site residue predictions combine several features in varying weights. Conservation and solvent accessibility seem to be the most discriminating factors. At the current state, an average of $70 \%$ accuracy is achieved by many independent studies.

\section{Supervised Learning is the Common Approach for Predic-} tion

Most interface residue predictive methods use neural networks or SVM to predict interface residues. A set of known positive (interface residue) and negative (surface) residue dataset is used to train machine learning algorithms. A residue is represented by a combination of features such as its type, frequency, etc. The choice of features mainly depends on availability of structure information.

\section{Need a Well Characterized "Gold Standard" Dataset}

Comparison of the current prediction methods with each other is not easy, because all of them use different datasets of differing sizes and content. Most of them use relatively small datasets that might not be sufficient to draw strong conclusions. Therefore, development of a "gold standard" complex dataset for interface residues would be very useful.

\section{Predicting Interactions Using Interfaces}

The next question is to find interacting residues between two protein chains. Docking has been extensively used to predict interface residues of two interacting proteins. However, docking at atomic details can be computationally a very demanding process [109-111]. Approximate docking ap- 
Table 1. Available Protein - Protein Interaction Prediction Web Servers

\begin{tabular}{|c|c|c|c|c|}
\hline Web Server & Availibility/truncate & Prediction Method & Binding Site Properties & Reference \\
\hline ProMate & $\begin{array}{l}\text { http://bioportal.weizmann.ac.il/ } \\
\text { promate }\end{array}$ & $\begin{array}{l}\text { Training on a dataset, } \\
\text { optimization of the pa- } \\
\text { rameters and prediction }\end{array}$ & $\begin{array}{l}\text { Amino acid propensities, pairwise amino acid } \\
\text { distribution, residue conservation, geometric } \\
\text { properties and other specific informations }\end{array}$ & [56] \\
\hline PINUP & $\begin{array}{l}\text { http://sparks.informatics.iupui.e } \\
\text { du/PINUP/ }\end{array}$ & $\begin{array}{l}\text { Empirical Scoring Func- } \\
\text { tion }\end{array}$ & $\begin{array}{l}\text { Side chain energy score, conservation score and } \\
\text { residue interface propensity }\end{array}$ & [103] \\
\hline PPI-Pred & $\begin{array}{l}\text { http://bioinformatics.leeds.ac.uk } \\
\text { /ppi-pred }\end{array}$ & SVM & $\begin{array}{l}\text { Surface shape, conservation, electrostatic poten- } \\
\text { tial, hydrophobicity, residue interface propensity, } \\
\text { solvent accessible surface area }\end{array}$ & {$[100]$} \\
\hline SPPIDER & http://sppider.cchmc.org/ & Neural Network & Solvent accessibility & {$[102]$} \\
\hline Meta-PPISP & $\begin{array}{l}\text { http://pipe.scs.fsu.edu/meta- } \\
\text { ppisp.html }\end{array}$ & Neural Network & $\begin{array}{c}\text { Combination of scores derived from other serves } \\
\text { i.e. ProMate, PINUP. }\end{array}$ & [108] \\
\hline PRISM & http://prism.ccbb.ku.edu.tr & Scoring Function & Structural matching & {$[72,115]$} \\
\hline SHARP & $\begin{array}{c}\text { http://www.bioinformatics.susse } \\
\text { x.ac.uk/SHARP2 }\end{array}$ & Patch Score Calculation & $\begin{array}{l}\text { Solvation potential, hydrophobicity, accessible } \\
\text { surface area, residue interface propensity, planar- } \\
\text { ity and protrusion }\end{array}$ & [118] \\
\hline cons-PPISP & $\begin{array}{c}\text { http://pipe.scs.fsu.edu/ppisp.htm } \\
1\end{array}$ & Neural Network & Sequence information and solvent accessibility & [98] \\
\hline
\end{tabular}

proaches (i.e., docking at coarser levels) have been investigated to be used in predicting interacting proteins and protein complexes. For example, PIPER [112] used fast fourier transform approach to docking to deal with the computational complexity. Structural superposition of interfaces combined with protein binding site prediction methods can be used to determine interactions overcoming the computationally expensive docking $[72,113]$. In addition to docking approaches, structural complementary can be exploited by machine learning based methods. Chung et al. [114] used a two-level approach, first predicting binding site residues, and then predicting interacting sites using a machine learning method.

In a recent study, we presented an efficient approach to predict protein-protein interfaces using a set of template interfaces [72, 115] for efficient docking of pairwise protein chains. The algorithm is a structure based method. It predicts interacting residues between two protein chains (in other words, predicts the interface between two chains) by structural matching and evolutionary similarities (structurally conserved hot spot residues). The algorithm seeks possible binary interactions between proteins (targets) through similar known interfaces. Template interfaces are the hot spot containing, biologically relevant protein interfaces extracted from PDB and the target set is the surfaces of the all proteins available in PDB. If surface region of two proteins are matching to complementary partners of a template interface (docking), then these two proteins are predicted as an interacting pair, and their binding site is the structurally matched residues (see Fig. (3)). The similarity between the template and target is measured with an empirical scoring function which considers the structural similarity (smaller the RMSD between template chain and target chain means better similarity) and the number of matched hot spot residues. The success of the algorithm [72] is shown by some case studies and verification of significant number of protein-protein interactions in DIP [116] and BIND [117].

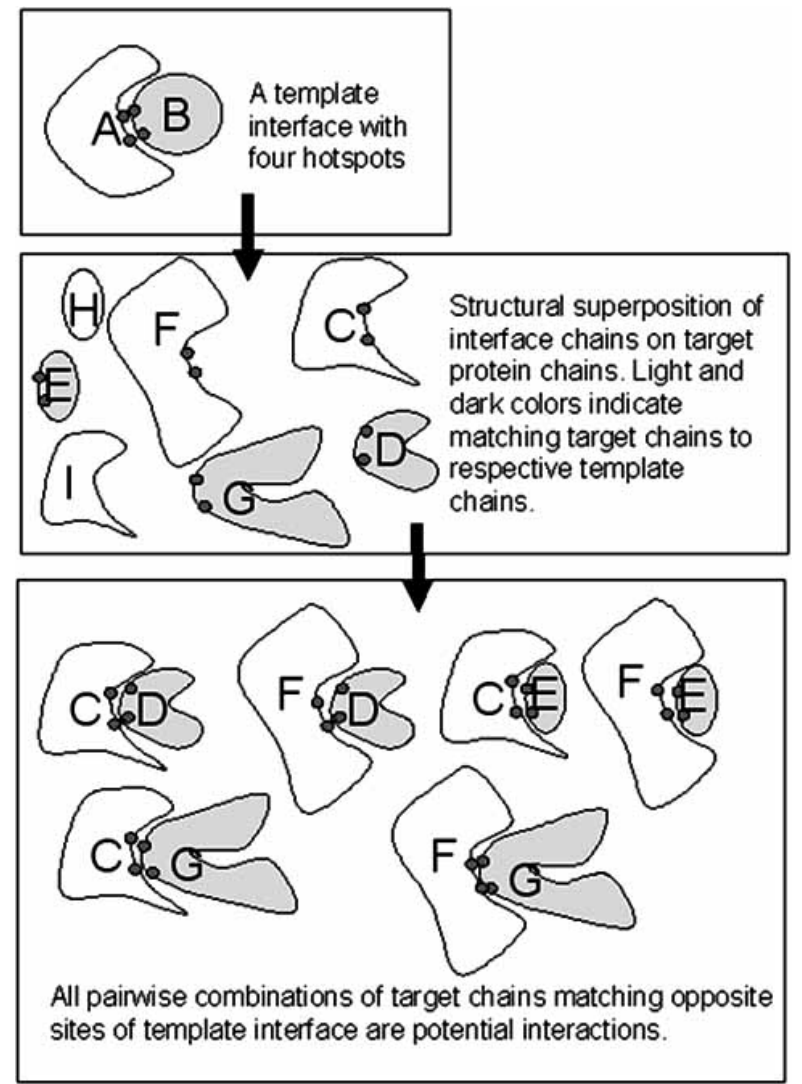

Fig. (3). The algorithm for protein interaction prediction using interfaces. 


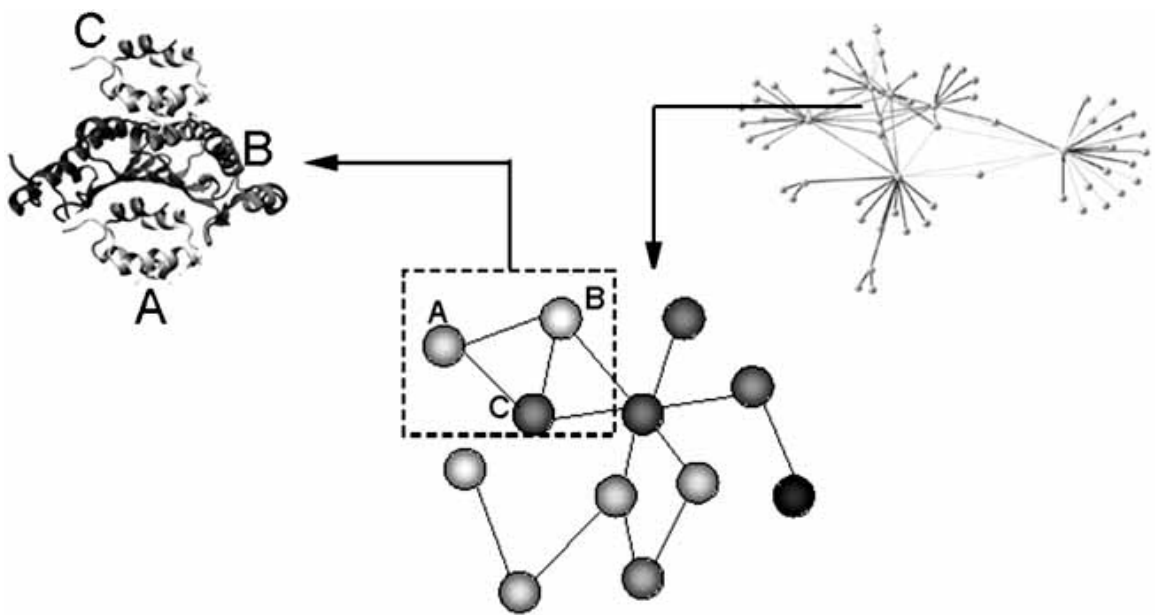

Fig. (4). From protein complexes to protein-protein interaction networks. Left panel shows the physical interactions between Proteins A-B and B-C. If structural knowledge is not known, Proteins A and C might be detected as interacting (middle panel). In a classical network representation (right panel), neither the transient/obligate interactions can be differentiated nor the knowledge about physically interacting proteins can be deduced.

\section{From Pairwise Interactions to Networks}

One of the primary objectives of the post-genomic era is the elucidation of the interactome in model cellular systems. The detailed knowledge of the full network of proteinprotein interactions, i.e., the distribution and the number of interactions as well as the presence of key nodes in these networks, is expected to provide new insights into the structures and properties of biological systems.

With the recent findings, we now know that in proteinprotein interaction networks, most proteins have only a few interactions, whereas, on the other hand, a small number of 'hubs' are highly connected [12]. Removal of such hub proteins is often lethal, which makes these proteins essential in the cell [13]. Further they connect many crucial cellular processes [84]. The availability of interactomes for many organisms will continue to provide understanding of the global organization of cellular processes. Still, these interactomes will lack structural and chemical characteristics of each interaction. With the help of these characteristics, a deeper understanding of the physical phenomena taking place in the cells can be appreciated.

For example, from a structural point of view, structure can help in understanding the roles of these hubs in the affinity of the interactions. In classical network models, each interaction is equally weighted and there is no differentiation between permanent and transient interactions. Another major problem with these large-scale network models is that two proteins that are observed to be connected in the network might be an outcome of the cooperativity. Protein A will interact with protein $\mathrm{B}$ if and only if protein $\mathrm{C}$ is bound to the latter protein. Or, sometimes, interactions shown are not real physical interactions but rather a third protein is needed to connect them [19]. With the data obtained from highthroughput experiments and limited power of classical network representations (Fig. (4)), right panel), it is not possible to differentiate such cases.

In an elegant study, Kim et al. [16] combined structural modeling with network analysis. They used the interfaces in protein surfaces and found that for two or more proteins in- teracting with a common partner protein, there are two possibilities: i) proteins can use the same interface on the partner (mutually exclusive). ii) or the two proteins can use different interfaces (simultaneously possible). They found that, most of the mutually exclusive interactions were transient, because they cannot occur at the same time. On the other hand, simultaneously possible interactions are enriched in permanent associations, connecting members of the same complex.

As the complete list of pairwise protein-protein interactions and the structures of the constituting proteins become available, it will be possible to construct reliable proteinprotein interaction networks with molecular details, such as the structural knowledge of the binding sites of the constituting proteins (as illustrated in Fig. (4)). Such approaches and novel representations integrating different levels of details (Fig. (4)) will enable inferring new knowledge which would be difficult if not possible otherwise. Further, since traditional representation of protein interaction networks signifies each interaction equally, combining structure with networks will provide better targets for drug design. These models with time series data will be able to explain the dynamic behavior of living systems.

\section{REFERENCES}

[1] Kleanthous, C. Protein-protein recognition, Oxford University Press: Oxford; New York 2000.

[2] Ito, T.; Chiba, T.; Ozawa, R.; Yoshida, M.; Hattori, M. and Sakaki, Y. (2001) Proc. Natl. Acad. Sci. U S A, 98, 4569-74.

[3] Uetz, P.; Giot, L.; Cagney, G.; Mansfield, T. A.; Judson, R. S.; Knight, J. R.; Lockshon, D.; Narayan, V.; Srinivasan, M.; Pochart, P.; Qureshi-Emili, A.; Li, Y.; Godwin, B.; Conover, D.; Kalbfleisch, T.; Vijayadamodar, G.; Yang, M.; Johnston, M.; Fields, S. and Rothberg, J. M. (2000) Nature, 403, 623-7.

[4] Ho, Y.; Gruhler, A.; Heilbut, A.; Bader, G. D.; Moore, L.; Adams, S. L.; Millar, A.; Taylor, P.; Bennett, K.; Boutilier, K.; Yang, L.; Wolting, C.; Donaldson, I.; Schandorff, S.; Shewnarane, J.; Vo, M.; Taggart, J.; Goudreault, M.; Muskat, B.; Alfarano, C.; Dewar, D.; Lin, Z.; Michalickova, K.; Willems, A. R.; Sassi, H.; Nielsen, P. A.; Rasmussen, K. J.; Andersen, J. R.; Johansen, L. E.; Hansen, L. H.; Jespersen, H.; Podtelejnikov, A.; Nielsen, E.; Crawford, J.; Poulsen, V.; Sorensen, B. D.; Matthiesen, J.; Hendrickson, R. C.; Gleeson, F.; Pawson, T.; Moran, M. F.; Durocher, D.; Mann, M.; Hogue, C. W.; Figeys, D. and Tyers, M. (2002) Nature, 415, 180-3. 
[5] Zhu, H.; Bilgin, M.; Bangham, R.; Hall, D.; Casamayor, A.; Bertone, P.; Lan, N.; Jansen, R.; Bidlingmaier, S.; Houfek, T.; Mitchell, T.; Miller, P.; Dean, R. A.; Gerstein, M. and Snyder, M. (2001) Science, 293, 2101-5.

[6] Koonin, E. V.; Wolf, Y. I. and Karev, G. P. (2002) Nature, 420, 218-23.

[7] von Mering, C.; Krause, R.; Snel, B.; Cornell, M.; Oliver, S. G.; Fields, S. and Bork, P. (2002) Nature, 417, 399-403.

[8] Schwikowski, B.; Uetz, P. and Fields, S. (2000) Nat. Biotechnol., 18, 1257-61.

[9] Tong, A. H.; Drees, B.; Nardelli, G.; Bader, G. D.; Brannetti, B.; Castagnoli, L.; Evangelista, M.; Ferracuti, S.; Nelson, B.; Paoluzi, S.; Quondam, M.; Zucconi, A.; Hogue, C. W.; Fields, S.; Boone, C. and Cesareni, G. (2002) Science, 295, 321-4.

[10] Yook, S. H.; Oltvai, Z. N. and Barabasi, A. L. (2004) Proteomics, 4, 928-42.

[11] Albert, R.; Jeong, H. and Barabasi, A. L. (2000) Nature, 406, $378-$ 82.

[12] Barabasi, A. L. and Albert, R. (1999) Science, 286, 509-12.

[13] Jeong, H.; Mason, S. P.; Barabasi, A. L. and Oltvai, Z. N. (2001) Nature, 411, 41-2.

[14] Gerdes, S. Y.; Scholle, M. D.; Campbell, J. W.; Balazsi, G.; Ravasz, E.; Daugherty, M. D.; Somera, A. L.; Kyrpides, N. C.; Anderson, I.; Gelfand, M. S.; Bhattacharya, A.; Kapatral, V.; D'Souza, M.; Baev, M. V.; Grechkin, Y.; Mseeh, F.; Fonstein, M. Y.; Overbeek, R.; Barabasi, A. L.; Oltvai, Z. N.; Osterman, A. L. (2003) J. Bacteriol., 185, 5673-84.

[15] Keskin, O. and Nussinov, R. (2007) Structure, 15, 341-54.

[16] Kim, P. M.; Lu, L. J.; Xia, Y. and Gerstein, M. B. (2006) Science, 314, 1938-41.

[17] Kim, W. K.; Henschel, A.; Winter, C. and Schroeder, M. (2006) PLoS. Comput. Biol, 2, e124.

[18] Shoemaker, B. A. and Panchenko, A. R. (2007) PLoS. Comput. Biol., 3, e43.

[19] Aloy, P. and Russell, R. B. (2006) Nat. Rev. Mol. Cell. Biol., 7, 188-97.

[20] Chakrabarti, P. and Janin, J. (2002) Proteins, 47, 334-43.

[21] Jones, S. and Thornton, J. M. (1996) Proc. Natl. Acad. Sci. U S A, 93, 13-20.

[22] Jones, S. and Thornton, J. M. (1997) J. Mol. Biol., 272, 121-32.

[23] Keskin, O.; Tsai, C. J.; Wolfson, H. and Nussinov, R. (2004) Protein Sci., 13, 1043-55.

[24] Li, X.; Keskin, O.; Ma, B.; Nussinov, R. and Liang, J. (2004) J. Mol. Biol., 344, 781-95.

[25] Tsai, C. J.; Lin, S. L.; Wolfson, H. J. and Nussinov, R. (1997) Protein Sci., 6, 53-64.

[26] Tsai, C. J.; Xu, D. and Nussinov, R. (1997) Protein Sci., 6, $1793-$ 805 .

[27] Bogan, A. A. and Thorn, K. S. (1998) J. Mol. Biol., 280, 1-9.

[28] Ma, B.; Elkayam, T.; Wolfson, H. and Nussinov, R. (2003) Proc. Natl. Acad. Sci. US A, 100, 5772-7.

[29] Winter, C.; Henschel, A.; Kim, W. K. and Schroeder, M. (2006) Nucleic Acids Res., 34, D310-4.

[30] Shoemaker, B. A.; Panchenko, A. R. and Bryant, S. H. (2006) Protein Sci., 15, 352-61.

[31] Gong, S.; Park, C.; Choi, H.; Ko, J.; Jang, I.; Lee, J.; Bolser, D. M.; Oh, D.; Kim, D. S. and Bhak, J. (2005) BMC Bioinformatics, 6, 207.

[32] Stein, A.; Russell, R. B. and Aloy, P. (2005) Nucleic Acids Res., 33, D413-7.

[33] Davis, F. P. and Sali, A. (2005) Bioinformatics, 21, 1901-7.

[34] Kundrotas, P. J. and Alexov, E. (2007) Nucleic Acids Res., 35, D575-9.

[35] Tuncbag, N.; Gursoy, A.; Guney, E.; Nussinov, R. and Keskin, O., (2008).

[36] Argos, P. (1988) Protein Eng, 2, 101-13.

[37] Chothia, C. and Janin, J. (1975) Nature, 256, 705-8.

[38] Clackson, T. and Wells, J. A. (1995) Science, 267, 383-6.

[39] Janin, J. and Chothia, C. (1990) J. Biol. Chem., 265, 16027-30.

[40] Keskin, O.; Ma, B. and Nussinov, R. (2005) J. Mol. Biol., 345, 1281-94.

[41] Keskin, O.; Ma, B.; Rogale, K.; Gunasekaran, K. and Nussinov, R. (2005) Phys. Biol., 2, S24-35.

[42] Keskin, O. and Nussinov, R. (2005) Protein Eng. Des. Sel., 18, $11-$ 24.

[43] Korn, A. P. and Burnett, R. M. (1991) Proteins, 9, 37-55.
[44] Lijnzaad, P.; Berendsen, H. J. and Argos, P. (1996) Proteins, 25, 389-97.

[45] Lo Conte, L.; Chothia, C. and Janin, J. (1999) J. Mol. Biol., 285, 2177-98.

[46] Sheinerman, F. B.; Norel, R. and Honig, B. (2000) Curr. Opin. Struct. Biol., 10, 153-9.

[47] Tsai, C. J.; Lin, S. L.; Wolfson, H. J. and Nussinov, R. (1996) J. Mol. Biol., 260, 604-20.

[48] Xu, W. and Regnier, F. E. (1998) J. Chromatogr. A., 828, 357-64.

[49] Bordner, A. J. and Abagyan, R. (2005) Proteins, 60, 353-66.

[50] Lawrence, M. C. and Colman, P. M. (1993) J. Mol. Biol., 234, 94650 .

[51] Sheinerman, F. B. and Honig, B. (2002) J. Mol. Biol., 318, 161-77.

[52] Jones, S. and Thornton, J. M. (1997) J. Mol. Biol., 272, 133-43.

[53] Larsen, T. A.; Olson, A. J. and Goodsell, D. S. (1998) Structure, 6, 421-7.

[54] Ofran, Y. and Rost, B. (2003) J. Mol. Biol., 325, 377-87.

[55] Jones, S. and Thornton, J. M. (1995) Prog. Biophys. Mol. Biol., 63, 31-65.

[56] Neuvirth, H.; Raz, R. and Schreiber, G. (2004) J. Mol. Biol., 338, 181-99.

[57] Caffrey, D. R.; Somaroo, S.; Hughes, J. D.; Mintseris, J. and Huang, E. S. (2004) Protein Sci., 13, 190-202.

[58] Grishin, N. V. and Phillips, M. A. (1994) Protein Sci., 3, 2455-8.

[59] Valdar, W. S. and Thornton, J. M. (2001) Proteins, 42, 108-24.

[60] Glaser, F.; Pupko, T.; Paz, I.; Bell, R. E.; Bechor-Shental, D.; Martz, E. and Ben-Tal, N. (2003) Bioinformatics, 19, 163-4.

[61] Glaser, F.; Rosenberg, Y.; Kessel, A.; Pupko, T. and Ben-Tal, N. (2005) Proteins, 58, 610-7.

[62] Pupko, T.; Bell, R. E.; Mayrose, I.; Glaser, F. and Ben-Tal, N. (2002) Bioinformatics, 18 Suppl 1, S71-7.

[63] Thorn, K. S. and Bogan, A. A. (2001) Bioinformatics, 17, 284-5.

[64] DeLano, W. L. (2002) Curr. Opin. Struct. Biol., 12, 14-20.

[65] Gao, Y.; Wang, R. and Lai, L. (2004) J. Mol. Model., 10, 44-54.

[66] Guerois, R.; Nielsen, J. E. Serrano, L. (2002) J. Mol Biol., 320, 369-87.

[67] Kortemme, T. and Baker, D. (2002) Proc. Natl. Acad. Sci. U S A, 99, 14116-21.

[68] Gonzalez-Ruiz, D. and Gohlke, H. (2006) Curr. Med. Chem., 13, 2607-25.

[69] Rajamani, D.; Thiel, S.; Vajda, S. and Camacho, C. J. (2004) Proc. Natl. Acad. Sci. USA, 101, 11287-92.

[70] Hu, Z.; Ma, B.; Wolfson, H. and Nussinov, R. (2000) Proteins, 39, $331-42$.

[71] Guney, E.; Tuncbag, N.; Keskin, O. and Gursoy, A. (2008) Nucleic Acids Res., 36, D662-6.

[72] Aytuna, A. S.; Gursoy, A. and Keskin, O. (2005) Bioinformatics, 21, 2850-5.

[73] Dundas, J.; Ouyang, Z.; Tseng, J.; Binkowski, A.; Turpaz, Y. and Liang, J. (2006) Nucleic Acids Res., 34, W116-8.

[74] Laskowski, R. A. (1995) J. Mol. Graph., 13, 323-30, 307-8.

[75] Liang, J.; Edelsbrunner, H. and Woodward, C. (1998) Protein Sci., 7, 1884-97.

[76] Brady, G. P., Jr. and Stouten, P. F. (2000) J. Comput. Aided. Mol. Des., 14, 383-401.

[77] Levitt, D. G. and Banaszak, L. J. (1992) J. Mol. Graph., 10, 22934.

[78] Weisel, M.; Proschak, E. and Schneider, G. (2007) Chem. Cent. J., $1,7$.

[79] An, J.; Totrov, M. and Abagyan, R. (2005) Mol. Cell. Proteomics, 4, 752-61.

[80] Cole, C. and Warwicker, J. (2002) Protein Sci., 11, 2860-70.

[81] Smith, G. R.; Sternberg, M. J. and Bates, P. A. (2005) J. Mol. Biol., 347, 1077-101.

[82] Yogurtcu, O. N.; Erdemli, B.; Turkay, M.; Nussinov, R. and Keskin, O. (2008) Biophysical. J., in press.

[83] Barabasi, A. L. and Oltvai, Z. N. (2004) Nat. Rev. Genet., 5, $101-$ 13.

[84] Han, J. D.; Bertin, N.; Hao, T.; Goldberg, D. S.; Berriz, G. F.; Zhang, L. V.; Dupuy, D.; Walhout, A. J.; Cusick, M. E.; Roth, F. P. and Vidal, M. (2004) Nature, 430, 88-93.

[85] Bullock, A. N.; Debreczeni, J. E.; Edwards, A. M.; Sundstrom, M. and Knapp, S. (2006) Proc. Natl. Acad Sci. U S A, 103, 7637-42.

[86] Kamura, T.; Maenaka, K.; Kotoshiba, S.; Matsumoto, M.; Kohda, D.; Conaway, R. C.; Conaway, J. W. and Nakayama, K. I. (2004) Genes Dev., 18, 3055-65. 
[87] Beckett, D. (2004) Biochemistry, 43, 7983-91.

[88] Berman, H. M.; Westbrook, J.; Feng, Z.; Gilliland, G.; Bhat, T. N.; Weissig, H.; Shindyalov, I. N. and Bourne, P. E. (2000) Nucleic Acids Res., 28, 235-42.

[89] Valdar, W. S. and Thornton, J. M. (2001) J. Mol Biol., 313, 399416.

[90] Elcock, A. H. and McCammon, J. A. (2001) Proc. Natl. Acad. Sci U S A, 98, 2990-4

[91] Mintseris, J. and Weng, Z. (2003) Proteins, 53, 629-39.

[92] Henrick, K. and Thornton, J. M. (1998) Trends Biochem Sci., 23, 358-61.

[93] Carugo, O. and Argos, P. (1997) Protein Sci., 6, 2261-3.

[94] Zhu, H.; Domingues, F. S.; Sommer, I. and Lengauer, T. (2006) BMC Bioinformatics, 7, 27.

[95] Res, I.; Mihalek, I. and Lichtarge, O. (2005) Bioinformatics, 21, 2496-501.

[96] Ofran, Y. and Rost, B. (2007) Bioinformatics, 23, e13-6.

[97] Kundrotas, P. and Alexov, E. (2007) Int. J. Biol. Macromol., 41, 615-23.

[98] Zhou, H. X. and Shan, Y. (2001) Proteins, 44, 336-43.

[99] Fariselli, P.; Pazos, F.; Valencia, A. and Casadio, R. (2002) Eur.J Biochem. 269, 1356-61.

[100] Bradford, J. R. and Westhead, D. R. (2005) Bioinformatics, 21, 1487-94.

[101] Chung, J. L.; Wang, W. and Bourne, P. E. (2006) Proteins, 62, 630-40.

[102] Porollo, A. and Meller, J. (2007) Proteins, 66, 630-45.

[103] Liang, S.; Zhang, C.; Liu, S. and Zhou, Y. (2006) Nucleic Acids Res., 34, 3698-707.

Received: January 10, 2008

Accepted: January 22, 2008
[104] Negi, S. S. and Braun, W. (2007) J. Mol. Model., 13, 1157-67.

[105] Hsu, C. M.; Chen, C. Y.; Liu, B. J.; Huang, C. C.; Laio, M. H.; Lin, C. C. and Wu, T. L. (2007) BMC Bioinformatics, 8 Suppl 5, S8

[106] Burgoyne, N. J. and Jackson, R. M. (2006) Bioinformatics, 22 1335-42.

[107] Bradford, J. R.; Needham, C. J.; Bulpitt, A. J. and Westhead, D. R. (2006) J. Mol Biol., 362, 365-86.

[108] Zhou, H. X. and Qin, S. (2007) Bioinformatics, 23, 2203-9.

[109] Halperin, I.; Wolfson, H. and Nussinov, R. (2004) Structure, 12 1027-38.

[110] Halperin, I.; Ma, B.; Wolfson, H. and Nussinov, R. (2002) Proteins, 47, 409-43.

[111] Schneidman-Duhovny, D.; Nussinov, R. and Wolfson, H. J. (2004) Curr. Med. Chem., 11, 91-107.

[112] Kozakov, D.; Brenke, R.; Comeau, S. R. and Vajda, S. (2006) Proteins, 65, 392-406.

[113] Cockell, S. J.; Oliva, B. and Jackson, R. M. (2007) Bioinformatics, 23, 573-81.

[114] Chung, J. L.; Wang, W. and Bourne, P. E.(2007) BMC Bioinformatics, 8, 223.

[115] Ogmen, U.; Keskin, O.; Aytuna, A. S.; Nussinov, R. and Gursoy, A. (2005) Nucleic Acids Res., 33, W331-6.

[116] Salwinski, L.; Miller, C. S.; Smith, A. J.; Pettit, F. K.; Bowie, J. U. and Eisenberg, D. (2004) Nucleic Acids Res., 32, D449-51.

[117] Bader, G. D.; Betel, D. and Hogue, C. W. (2003) Nucleic Acids Res., 31, 248-50.

[118] Murakami, Y. and Jones, S. (2006) Bioinformatics, 22, 1794-5. 